\title{
TWISTED PROPERTIES OF BANACH SPACES
}

\author{
JESÚS M. F. CASTILLO, MANUEL GONZÁLEZ, \\ ANATOLIJ M. PLICHKO and DAVID YOST
}

\begin{abstract}
If $\mathscr{P}, \mathscr{Q}$ are two linear topological properties, say that a Banach space $X$ has the property $\mathscr{P}$-by$\mathscr{Q}$ (or is a $\mathscr{P}$-by- $\mathscr{Q}$ space) if $X$ has a subspace $Y$ with property $\mathscr{P}$ such that the corresponding quotient $X / Y$ has property $\mathscr{Q}$. The choices $\mathscr{P}, \mathscr{Q} \in\{$ separable, reflexive $\}$ lead naturally to some new results and new proofs of old results concerning weakly compactly generated Banach spaces. For example, every extension of a subspace of $L_{1}(0,1)$ by a WCG space is WCG. They also give a simple new example of a Banach space property which is not a 3-space property but whose dual is a 3 -space property.
\end{abstract}

\section{Introduction}

Throughout, $Y$ will be a closed subspace of a Banach space $X$ and $Z$ will be the corresponding quotient $X / Y$. Denote by $i$ and $q$ the inclusion and quotient mappings. In general, $Y$ need not be complemented in $X$, i.e. there need not be any continuous linear projection from $X$ onto $Y$.

By Banach space property we mean simply a property which is invariant under isomorphisms; it need not pass to subspaces or quotients, or even be possessed by finite-dimensional spaces. Say that $\mathscr{P}$ is a three-space property if whenever $Y$ and $Z$ have $\mathscr{P}$ then $X$ also has $\mathscr{P}$. For instance, separability is easily checked to be a 3 -space property. This notion becomes interesting when one realises that being isomorphic to a Hilbert space is not a 3-space property [17]. Given a property $\mathscr{P}$ we say, following [29], that $X$ has $\mathscr{P}_{*}$ if $X^{*}$ has $\mathscr{P}$. We prefer to write the star as a subscript to emphasize the fact that such an $X$ is the predual of a space with $\mathscr{P}$. Similarly we say that $X$ has $\mathscr{P}^{\text {co }}$ if $X^{* *} / X$ has $\mathscr{P}$. It is straightforward to prove that if $\mathscr{P}$ is a 3 -space property then $\mathscr{P}_{*}$ is also a 3-space property. For $\mathscr{P}^{\mathrm{co}}$ the same assertion follows from Lemma 2.1 below. Jarchow [29, §3, Remark 5(b)], asked whether the reverse implication for $\mathscr{P}_{*}$ is true; we shall see in $\S 4$ that it is not. It is not known to us if $\mathscr{P}$ must be a 3 -space property whenever $\mathscr{P}^{\mathrm{co}}$ is a 3-space property.

Since reflexivity is simply the property 0 -dimensional ${ }^{\mathrm{co}}$, this observation also establishes the well known fact that reflexivity is a 3-space property.

Received December 1, 1998. 
A group $G$ is called a $\mathscr{P}$-by- $\mathscr{Q}$ group if it contains a normal subgroup $N$ such that $N$ has property $\mathscr{P}$ and $G / N$ has $\mathscr{Q}$. As far as we know, this terminology first slipped into the literature in [23, p. 424]. It has been standard in group theory ever since [20] and we will use it here. Thus, given two Banach space properties $\mathscr{P}$ and $\mathscr{Q}$, we say that a Banach space $X$ has the property $\mathscr{P}$-by- $\mathscr{Q}$ if it admits a closed subspace $Y$ with property $\mathscr{P}$ such that $X / Y$ has $\mathscr{Q}$. It is an old idea that a complicated Banach space might "factor" through a nice subspace with a nice quotient. Our point is that the nice properties of the subspace and the quotient need not be the same. By analogy with twisted sums of Banach spaces [34] we will refer to such properties as twisted properties. Many of our results, e.g. Proposition 2.4, would be cumbersome to prove without this shorthand.

REMARK 1.1. Given this convention, $\mathscr{P}$ is a 3 -space property if and only if $\mathscr{P}$-by- $\mathscr{P}$ implies $\mathscr{P}$. It is very easy to check that "-by-" is an associative operation on the collection of all Banach space properties. That is, $\left(\mathscr{P}_{1}\right.$-by- $\left.\mathscr{P}_{2}\right)$-by$\mathscr{P}_{3}$ is exactly the same property as $\mathscr{P}_{1}$-by- $\left(\mathscr{P}_{2}\right.$-by- $\left.\mathscr{P}_{3}\right)$ and the parentheses can be omitted.

However "-by-" is not an associative operation for groups. If $\mathscr{P}_{n}$ is the property "cyclic of order $n$ ", then the alternating group $A_{4}$ is $\left(\mathscr{P}_{2}\right.$-by- $\left.\mathscr{P}_{2}\right)$-by$\mathscr{P}_{3}$, but not $\mathscr{P}_{2}$-by- $\left(\mathscr{P}_{2}\right.$-by- $\left.\mathscr{P}_{3}\right)$.

In $\S 2$, we present a number of elementary results. Since WCG (weakly compactly generated) spaces form a natural class which includes all separable and reflexive spaces, we exhibit some basic results about them in §3. Johnson and Lindenstrauss [32] showed that every WCG-by-separable space is WCG. We show in $\S 3$ that this result is best possible in the sense that separability cannot be replaced by any other property. They also showed that reflexive-byWCG implies WCG. We give a new elementary proof of this, and then recall their example of a separable-by-reflexive space which is not WCG.

This leads directly, in $\S 4$, to the counterexample mentioned in the abstract; the property reflexive-by-separable is not a 3 -space property, but the dual property is.

Section 5 consists mainly of known results, relating to projections on WCG spaces, which are needed later, some of them with new proofs. The main part of this paper is probably $\S 6$ and $\S 7$, where we present a new family of stability results (all of which depend on the fact that (reflexive-by-separable) $)_{*}$ is a 3space property) and some open problems. For conciseness, let us say that $Y$ has the property $\mathscr{E} \mathscr{P}$ if $Y$-by- $\mathscr{P}$ implies $\mathscr{P}$, i.e. whenever $X$ is a Banach space containing $Y$ and the quotient space $X / Y$ has property $\mathscr{P}$, then $X$ also has $\mathscr{P}$. The observation that $\mathscr{E} \mathscr{P}$ is always a 3 -space property (even when $\mathscr{P}$ is not) simplifies several arguments. We show that many non-reflexive spaces have 
$\mathscr{E} \mathrm{WCG}$, in particular all subspaces of $L_{1}(0,1)$ or the James Tree space. In $\S 8$ the related class of separably distinguished spaces is briefly discussed.

\section{Basic results}

The following result, which appeared first in [9], is a basic tool. It seems worthwhile to give a succinct proof. Recall that $Y$ is a closed subspace of the Banach space $X$.

LEMMA 2.1.

(i) The subspace $X+Y^{\perp \perp}$ is always closed in $X^{* *}$,

(ii) there is a natural isomorphism

$$
\frac{(X / Y)^{* *}}{X / Y} \cong \frac{X^{* *}}{X+Y^{\perp \perp}},
$$

(iii) and another isomorphism $Y^{* *} / Y \cong\left(X+Y^{\perp \perp}\right) / X$.

Proof. Let $H:(X / Y)^{* *} \rightarrow(X / Y)^{* *} /(X / Y)$ be the obvious natural mapping. Then

$\operatorname{ker}\left(H q^{* *}\right)=\left(q^{* *}\right)^{-1}(\operatorname{ker} H)=\left(q^{* *}\right)^{-1}\left(q^{* *} X\right)=X+\operatorname{ker} q^{* *}=X+Y^{\perp \perp}$.

This proves (i). Since the range of $H q^{* *}$ is the Banach space $(X / Y)^{* *} /(X / Y)$, (ii) follows easily. Finally, considering the bitranspose of the inclusion $Y \subset X$, one has

$$
Y^{* *} / Y \cong Y^{\perp \perp} /\left(X \cap Y^{\perp \perp}\right) \cong\left(Y^{\perp \perp}+X\right) / X
$$

so (iii) is also established.

LeMma 2.2. For any two Banach space properties $\mathscr{P}$ and $\mathscr{Q}, \mathscr{P}_{*}-$ by- $\mathscr{Q}_{*}$

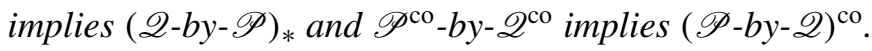

Given two properties $\mathscr{P}$ and $\mathscr{Q}$, say that a Banach space $X$ has the property $\mathscr{P} \oplus \mathscr{Q}$ if it can be decomposed as $X=Y \oplus Z$ where $Y$ has property $\mathscr{P}$ and $Z$ has property $\mathscr{Q}$. The next result is also easy to prove.

Proposition 2.3. Let $\mathscr{P}$ and $\mathscr{Q}$ be two 3 -space properties which are enjoyed by the trivial space $\{0\}$. Then $\mathscr{P} \oplus \mathscr{Q}$ is a 3 -space property if and only if $\mathscr{P}$ -

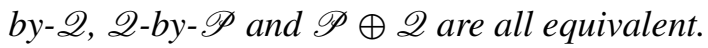

The usefulness of the next result far exceeds the difficulty of its proof.

Proposition 2.4. Let $\mathscr{P}$ and $\mathscr{Q}$ be any 3 -space properties. If $\mathscr{Q}$-by- $\mathscr{P}$

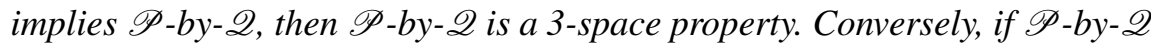
is a 3 -space property and $\mathscr{P}$ and $\mathscr{Q}$ are enjoyed by the trivial space $\{0\}$, then

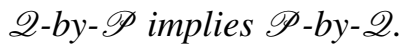


Proof. Necessity is trivial. Sufficiency follows immediately from associativity:

$$
\mathscr{P} \text {-by- } \mathscr{Q} \text {-by- } \mathscr{P} \text {-by- } \mathscr{Q} \Rightarrow \mathscr{P} \text {-by- } \mathscr{P} \text {-by- } \mathscr{Q} \text {-by- } \mathscr{Q} \Rightarrow \mathscr{P} \text {-by- } \mathscr{Q}
$$

Call a property $\mathscr{P}$ liftable if it satisfies the condition: whenever $X / Y$ has $\mathscr{P}$, then there is some subspace $M$ of $X$ having $\mathscr{P}$ such that $M+Y=X$.

Proposition 2.5. Let $\mathscr{P}$ and $\mathscr{Q}$ be 3 -space properties. Assume that $\mathscr{Q}$ passes to quotients and that $\mathscr{P}$ is liftable. Then $\mathscr{P}$-by-Q2 is a 3-space property.

Proof. It clearly suffices to show that $\mathscr{Q}$-by- $\mathscr{P}$ implies $\mathscr{P}$-by- $\mathscr{Q}$, so suppose that $Y$ has $\mathscr{Q}$ and $X / Y$ has $\mathscr{P}$. By hypothesis, there is a subspace $M$ of $X$ having property $\mathscr{P}$ such that $X=Y+M$. To finish the proof it is only necessary to verify that $X / M$ has $\mathscr{Q}$. Since $\mathscr{Q}$ passes to quotients, this is a consequence of the isomorphism $X / M=(M+Y) / M \cong Y /(Y \cap M)$.

One candidate for a property dual to liftability is the following: say that a property $\mathscr{Q}$ is extendable if it satisfies the condition: whenever $Y \subset X$ and $Y$ has $\mathscr{Q}$, then $X$ contains a closed subspace $M$ such that $M \cap Y=\{0\}, M+Y$ is closed (which of course implies that $Y$ is isomorphic to a subspace of $X / M$ ) and $X / M$ has $\mathscr{Q}$. We leave the following result as an exercise.

Proposition 2. 5*, Let $\mathscr{P}$ and $\mathscr{Q}$ be 3 -space properties. Assume that $\mathscr{P}$ passes to subspaces and that $\mathscr{Q}$ is extendable. Then $\mathscr{P}$-by- $\mathscr{Q}$ is a 3-space property.

It is clear that finite-dimensionality is both liftable and extendable. However separability is not extendable, as the following argument shows. If it were, $\ell_{\infty}$ would contain a closed subspace $M$ with $c_{0} \cap M=\{0\}, c_{0}+M$ closed, and $\ell_{\infty} / M$ separable. Obviously $c_{0}$ could then be identified with a subspace of $\ell_{\infty} / M$, which by Sobczyk's Theorem (e.g. [28]) must be complemented. Composing this projection with the quotient map $\ell_{\infty} \rightarrow \ell_{\infty} / M$ would then give us a projection $\ell_{\infty} \rightarrow c_{0}$, a well known impossibility. We note in passing the old problem: does every infinite-dimensional Banach space have an infinitedimensional separable quotient space?

The next result says that separability is a liftable property. This appears implicitly in [52, Corollary 6], and explicitly in [54, Lemma 2].

Lemma 2.6. Let $X$ be any Banach space, $Y$ a closed subspace.

(i) If $X / Y$ is separable, then $X=M+Y$ for some separable subspace $M$ of $X$.

(ii) If $S_{0}$ is any separable subspace of $X$, one can find a separable subspace $S$ containing $S_{0}$, for which $Y+S$ is closed. 
Proof. (i) Let $\varphi: X \rightarrow X / Y$ be the quotient map, and choose a countable set $C$ in the open unit ball of $X$ so that $\varphi(C)$ is dense in the open unit ball of $X / Y$. Let $M$ be the closed linear span of $C$, and $U$ the open unit ball of $M$. Then $\varphi(U)$ is a dense subset of the unit ball. Furthermore $\varphi(U)$ is closed under the formation of infinite convex combinations, because $U$ has the same property. This implies that $\varphi(U)$ contains the interior of its closure. (In the language of [28, §22], CS-compact sets are semi-closed. This is the basic technical fact which leads to the open mapping and closed graph theorems.) Thus $\varphi(U)$ must contain the open unit ball of $X / Y$. It follows that $\varphi(M)=X / Y$, i.e. that $Y+M=X$. Of course $M$ is a separable subpace of $X$.

(ii) Clearly $\overline{Y+S_{0}} / Y$ is separable, so (i) allows us to choose some separable subspace $S$ of $X$ such that $Y+S=\overline{Y+S_{0}}$. One may assume that $S$ contains $S_{0}$, replacing $S$ by $\overline{S+S_{0}}$ if necessary.

With the same proof, Lemma 2.6 remains valid for arbitrary density characters. An easy corollary is [57, Theorem 2]: if $X / Y$ is separable, then $X$ is isomorphic to a quotient of $Y \oplus M$, for some separable space $M$.

Thus if $\mathscr{Q}$ is any 3 -space property which passes to quotients, then being separable-by- $\mathscr{Q}$ is a 3 -space property. In particular, separable-by-Asplund, separable-by-(not containing $\left.\ell_{1}\right)$, separable-by-(Banach-Saks) and separableby-(B-convex) are 3-space properties. Even more is true.

THEOREM 2.7. Let $\mathscr{Q}$ be any 3-space property which passes to quotients. Then being separable-by-Q is a 3-space property which passes to quotients. If in addition $\mathscr{Q}$ passes to subspaces, then separable-by- $\mathscr{Q}$ also passes to subspaces.

Proof. It is abundantly clear by now that being separable-by- $\mathscr{Q}$ is a 3 -space property. For the other two parts, let $X$ be a separable-by- $\mathscr{Q}$ Banach space, $Y$ an arbitrary subspace. Then $X$ has a separable subspace $S_{0}$ so that $X / S_{0}$ has $\mathscr{Q}$. Let $S$ be given by Lemma 2.6(ii). Since $Y+S$ is closed, one has

$$
\frac{X / Y}{(Y+S) / Y} \cong \frac{X}{Y+S} \cong \frac{X / S_{0}}{(Y+S) / S_{0}}
$$

which obviously has $\mathscr{Q}$. Since $(Y+S) / Y$ is separable, the quotient $X / Y$ is separable-by- $\mathscr{Q}$.

Now suppose that $\mathscr{Q}$ passes to subspaces. Since $X / S$ is a quotient of $X / S_{0}$, any subspace of $X / S$ has $\mathscr{Q}$. In particular $Y /(S \cap Y) \cong(Y+S) / S \subset X / S$ has $\mathscr{Q}$. Since $S \cap Y$ is separable, it follows that $Y$ has separable-by- $\mathscr{Q}$.

In particular, being separable-by-reflexive is a 3-space property which passes to subspaces and quotients. We single out this property because it will be of considerable interest to us. It will be shown later that reflexive-by-separable 
implies separable $\oplus$ reflexive. However reflexivity is not a liftable property, as shown at the end of the next section. Nevertheless, there are other liftable properties.

For a second application, recall that $\ell_{1}$ has the so-called lifting property, i.e. whenever it is isomorphic to a quotient space $X / Y$ then $Y$ is complemented and so $X$ is isomorphic to $\ell_{1} \oplus Y$. Thus, being isomorphic to $\ell_{1}$ is a liftable property. Similarly, being isomorphic to $\ell_{\infty}$ is an extendable property, as is being injective (i.e. being complemented in every superspace).

Proposition 2.8. Let $\mathscr{P}$ be a 3-space property. Then $\ell_{1}$-by- $\mathscr{P}$ is a 3-space property.

Of course $\ell_{1}$ can be replaced by $\ell_{1}(\Gamma)$ for any $\Gamma$ in this result. It is natural to ask if $\ell_{1}$ can also be replaced by $L_{1}$ or by an $\mathscr{L}_{1}$ space. One has the following partial result.

For conciseness, let us say that a Banach space has the property $\mathscr{C} \mathscr{B}$ if it is complemented in its own bidual.

Proposition 2.9. Let $\mathscr{P}$ be a 3-space property which implies $\mathscr{C} \mathscr{B}$. Then $\mathscr{L}_{1}$-by- $\mathscr{P}$ is a 3-space property.

Proof. It suffices to show that $\mathscr{P}$-by- $\mathscr{L}_{1}$ implies $\mathscr{L}_{1} \oplus \mathscr{P}$ and this follows from the well known result of Lindenstrauss [36, Lemma 1], that if $Y$ has $\mathscr{C} \mathscr{B}$ and $Z$ is an $\mathscr{L}_{1}$ space, then $Y$ is complemented in $X$.

In particular $\mathscr{L}_{1}$-by-injective, $\mathscr{L}_{1}$-by-quasireflexive etc. are 3 -space properties. Now fix a non-degenerate measure $\mu$ and let $\mathscr{L}$ be the property "isomorphic to $L_{1}(\mu)$ ". Since $L_{1}(\mu)$ is isomorphic to its square, the preceding argument shows that, under the same hypotheses, $\mathscr{L}$-by- $\mathscr{P}$ is a 3 -space property.

If $\mathscr{C} \mathscr{B}$ were known to be a 3 -space property, the previous result would have a simpler formulation. A counterexample to this problem has recently been found [5, Theorem 1], so the following partial result may be of interest. It can also be proved by diagram chasing.

Proposition 2.10. Every $\mathscr{C} \mathscr{B}$-by-reflexive space is $\mathscr{C} \mathscr{B}$.

Proof. If $Z$ is reflexive, then $X^{* *}=X+Y^{\perp \perp}$ by Lemma 2.1. If $Y$ is complemented in its bidual, one can write $Y^{\perp \perp}=Y \oplus M$ for some closed subspace $M$. Then $X+M=X+Y+M=X+Y^{\perp \perp}=X^{* *}$ and $X \cap M=$ $X \cap Y^{\perp \perp} \cap M=Y \cap M=\{0\}$. Thus $X^{* *}=X \oplus M$. 


\section{Weakly compactly generated spaces for beginners}

Consider now the following generalization of separability and reflexivity: a Banach space $X$ is said to be weakly compactly generated (WCG) if it contains a weakly compact subset having dense span. Apart from separable and reflexive spaces, other popular examples are $c_{0}(\Gamma)$, for any set $\Gamma\left(\left\{e_{\gamma}: \gamma \in \Gamma\right\} \cup\{0\}\right.$ is weakly compact), $L_{1}(\mu)$ for any $\sigma$-finite measure $\mu$ (if $\mu$ is finite, the inclusion mapping $L_{2}(\mu) \rightarrow L_{1}(\mu)$ is continuous and has dense range) and the spaces $C(K)$ when $K$ is a weakly compact subset of a Banach space ([1, p. 36], or [60, p. 189]).

It is clear that any quotient (in particular, any complemented subspace) of a WCG space is WCG. It is hard to find subspaces of WCG spaces which are not WCG, but both Rosenthal [49] and Argyros, [2] or [18, §1.6], succeeded in doing so. In this section we concentrate on the question: what can be said about $\mathscr{P}$-by- $\mathscr{Q}$ spaces when $\mathscr{P}, \mathscr{Q} \in$ sseparable, reflexive, WCG $\}$ ? Of course all three properties pass to (finite) direct sums. As various examples show, being WCG is not a 3 -space property.

This section begins with two results which can be proved in an elementary manner. They are due to Johnson and Lindenstrauss [32]. The original proof of the first required detailed knowledge of projections in WCG spaces. We give a new proof, using only the elementary lifting argument from [19].

Proposition 3.1. Every reflexive-by-WCG space is WCG.

Proof. Let $Y$ be a reflexive subspace of $X$ and let $K$ be a weakly compact set in $X / Y$ spanning a dense subspace. Take a bounded subset $A$ in $X$ such that $q(A)=K$, and denote by $\bar{B}$ the weak* closure of a set $B$ in $X^{* *}$. Clearly $q^{* *} \mid X=q$ and $q^{* *}$ is weak ${ }^{*}$ continuous, and so $q^{* *}(\bar{A}) \subseteq \overline{q^{* *}(A)}=\overline{q(A)}=$ $K \subset X / Y=q^{* *}(X)$. Since $Y$ is reflexive, one has $Y^{\perp \perp}=Y \subset X$ and so $\bar{A} \subseteq X+\operatorname{ker} q^{* *}=X+Y^{\perp \perp}=X$. Thus $A$ is relatively weakly compact, and the relatively weakly compact set $A \cup \operatorname{ball}(Y)$ spans a dense subspace of $X$.

Observe that the lifting of weakly compact sets is not always possible: denoting by $J L$ the space from Example 3.6, weakly compact sets in $J L$ are separable while $J L / c_{0}$ admits nonseparable weakly compact sets (e.g. its unit ball!), and these cannot be lifted.

As mentioned in the introduction, the hypothesis of reflexivity in Proposition 3.1 can be weakened considerably. This is the subject of $\$ 6$ and $\$ 7$.

Proposition 3.2. Every WCG-by-separable space is WCG.

Proof. Suppose that $Y$ is WCG and $X / Y$ is separable. By Lemma 2.6 (i), $X=Y+M$, where $M$ is separable. Obviously the union of a norm compact 
set which generates $M$ and a weakly compact set which generates $Y$ will do the trick.

Johnson and Lindenstrauss also proved a useful converse to Proposition 3.2. Namely, if $X$ is WCG and $X / Y$ is separable, then $Y$ is WCG. This result lies a little deeper, and will be proved in $\S 5$.

Theorem 3.4 below shows that if $\mathscr{P}$ is any property for which WCG-by- $\mathscr{P}$ implies WCG, then $\mathscr{P}$ implies separability. Thus Proposition 3.2 is "best possible". To prove that, we need the following well known result, exact attribution of which is a little difficult. It is an obvious consequence of [40, Theorem 6]. The earliest explicit statement which we could find was [48, p. 19, footnote].

EXAMPLE 3.3. If $\Gamma$ is a set of cardinality equal to the continuum, then there is a subspace $A$ of $\ell_{\infty}$ containing $c_{0}$ so that $A / c_{0} \cong c_{0}(\Gamma)$.

Since every weakly compact subset of $\ell_{\infty}$ is separable, it is clear that $A$ is not WCG. In particular, $c_{0}$ is uncomplemented in $A$. (The existence of a non-trivial extension of $c_{0}$ by $c_{0}(\Gamma)$, for any uncountable set $\Gamma$, follows easily from this.) Thus one has a counterexample to the 3-space problem for WCG spaces. Although this is the simplest counterexample, it was not the first to be discovered. For a brief discussion of some others see [60, p. 191].

THEOREM 3.4. Let $Z$ be any non-separable Banach space. Then there is a non-WCG extension of $c_{0}$ by $Z$.

Proof. We assume that $Z$ is WCG, since the result is trivial otherwise. According to Reif [47, pp. 336-7], $Z$ admits a Markuševič basis $\left(x_{\gamma}, f_{\gamma}\right)_{\gamma \in \Gamma}$, i.e. a biorthogonal system in $Z \times Z^{*}$ for which $\left(x_{\gamma}\right)_{\gamma \in \Gamma}$ separates the points of $Z^{*}$ and $\left(f_{\gamma}\right)_{\gamma \in \Gamma}$ separates the points of $Z$. One may assume without loss of generality that $\left(f_{\gamma}\right)$ is bounded. Then the map $T: Z \rightarrow c_{0}(\Gamma)$ defined by $T x=\left(f_{\gamma}(x)\right)$ is easily checked to be a bounded linear operator with dense range. Let $A$ be the space defined in Example 3.3 and let $Q: A \rightarrow c_{0}(\Gamma)$ be the quotient mapping.

Set $B=\{x \in A:\|x\| \leq 1, Q x \in T$ (ball $Z$ ) $\}$, and let $X$ be the linear span of $B$, equipped with the norm whose unit ball is $B$. It is routine to check that $X$ is a Banach space, and that $B \cap c_{0}=\operatorname{ball}\left(c_{0}\right)$. So $X$ contains $c_{0}$ as a subspace and it is easily verified that $X / c_{0} \cong Z$. However the identity mapping $X \rightarrow A$ is continuous and has dense range, so $X$ cannot be WCG. In particular, this copy of $c_{0}$ cannot be complemented.

The idea of the preceding proof can be found in [32, Example 1], [60, Lemma] and [57, Theorem 1]. The same argument appears again in 6.2. In categorical language, $X$ is simply the pullback of $T$ and $Q$. For more applications of this sort of argument, see [5] and [6]. 
Theorem 3.4 lies rather deep, because Reif's result depends on results about projections in WCG spaces. (These are discussed briefly in §5.) Not surprisingly, special cases have easier proofs. We mention just two.

Example 3.5. There is an extension of $c_{0}$ by a WCG $\mathscr{L}_{1}$ space which is not WCG.

Proof. Denote by 2 the two point measure space $\{-1,1\}$, with each point having measure $\frac{1}{2}$. Let $\mu$ be the product measure on $S=\mathbf{2}^{\Gamma}$, and denote by $p_{\gamma}: S \rightarrow \mathbf{2}$ the projection onto the $\gamma^{\text {th }}$ coordinate. Define $T: L_{1}(\mu) \rightarrow$ $c_{0}(\Gamma)$ by $(T f)(\gamma)=\int_{S} f p_{\gamma} d \mu$. (This is well defined since the characteristic function of any elementary measurable set gets mapped to a finitely supported function.) Then $T$ is a bounded linear operator with dense range and one proceeds as before.

The literature does not seem to contain many examples of non-trivial twisted sums of a $\mathscr{L}_{\infty}$ and a $\mathscr{L}_{1}$ space. For further discussion of this, see [6]. We have recently found a separable example, details of which will appear elsewhere.

Since the inclusion mapping $\ell_{p}(\Gamma) \rightarrow c_{0}(\Gamma)$ is continuous and has dense range, one may conclude that there is a non-trivial twisted sum of $c_{0}$ and $\ell_{p}(\Gamma)$ for $1<p<\infty$. In particular, Johnson and Lindenstrauss [32, Example 1] first found the following.

Example 3.6. There is an extension of $c_{0}$ by a Hilbert space, denoted in the rest of this paper by $J L$, which is not WCG.

In particular, $J L$ is not reflexive $\oplus$ separable, and $c_{0}$ is an uncomplemented subspace. It is worth noting that the natural mapping $J L \rightarrow A$ is injective. Here $A$ is again the space from Example 3.3. It follows that every weakly compact subset of $J L$ embeds in $A$ and so is separable. Thus every reflexive subspace of $J L$ is separable. Similarly $J L^{*}$ is weak* separable. The reader can find further properties of $J L$ in the original paper [32, Example 1], or in the survey [60, Example 3].

The lifting property of $\ell_{1}$ implies that the dual space $J L^{*}$ is isomorphic to the direct sum of $\ell_{1}$ and a Hilbert space, and thus $J L^{*}$ is weakly compactly generated. This was the first example to show that $\mathrm{WCG}_{*}$ does not imply WCG.

Theorem 3.4 shows that any property, which implies WCG but does not imply separability, is not liftable. Amongst such properties are reflexivity, being isomorphic to $c_{0}(\Gamma)$ and admitting a non-separable weakly compact subset. 


\section{Asplund spaces and the duality counterexample}

Jarchow $[29, \S 3$, Remark 5(b)] asked whether a Banach space property $\mathscr{P}$ has to be a 3 -space property whenever $\mathscr{P}_{*}$ is a 3 -space property. The answer to this question is already known (although not very well). It is implicit in [7] and explicit in $[8, \S 6.6]$ that the hereditary Dunford-Pettis property provides a counterexample. In [12, Example III-10(3)], it is shown that (WCG \& Asplund) furnishes another counterexample. Whether the property WCG itself provides a counterexample is still open; see $\$ 5$ and $\S 7$ for some discussion of this problem. We give here a much simpler counterexample. Specifically, we will show that reflexive-by-separable is not a 3-space property, but that (reflexiveby-separable $)_{*}$ is a 3 -space property. Another counterexample, having Čech complete unit ball, is discussed briefly in $\S 8$.

Let us say that $X$ has the property $\mathscr{P}$ sep whenever every separable subspace of $X$ has $\mathscr{P}$. A property is said to be separably determined if $\mathscr{P}$ and $\mathscr{P}$ sep are the same. For example, reflexivity is separably determined. Also, Theorem 4.1 tells us that if $\mathscr{P}$ is a 3 -space property which passes to subspaces, then $\mathscr{P}$ sep is also a 3 -space property.

Another useful notion is that of Asplund space. We will call a Banach space an Asplund space if every separable subspace has separable dual. Symbolically, being Asplund is the property $\left(\text { separable }_{*}\right)^{\text {sep }}$. See [43, §2] or [59] for some equivalent formulations in terms of automatic differentiability of convex functions, or the dual formulation in the next paragraph.

Although it is not essential at this stage, recall that a Banach space is said to have the Radon-Nikodým property (RNP) if every bounded subset admits a slice (i.e. a non-empty intersection with a half-space) of arbitrarily small diameter. There are many other equivalent formulations: the reader may refer to [52] or [15] for further enlightenment. Amongst other things, Asplund spaces coincide with spaces whose duals have RNP. In particular, separable dual spaces have the Radon-Nikodým Property.

Since the RNP is a 3 -space property ([15, p. 211] or [8, Chapter 6]), the same occurs with the $\mathrm{RNP}_{*}$. We prefer a direct proof.

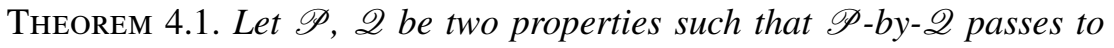
subspaces. Then $\mathscr{P}^{\mathrm{sep}}$-by- $\mathscr{Q}^{\mathrm{sep}}$ implies $(\mathscr{P} \text {-by- } \mathscr{Q})^{\mathrm{sep}}$.

Proof. Let $X$ be a Banach space, $Y$ a subspace with $\mathscr{P}$ sep so that $X / Y$ has $\mathscr{Q}^{\text {sep }}$. Let $S_{0}$ be any separable subspace of $X$. By Lemma 2.6(ii), there is a separable subspace $S$ of $X$ such that $Y+S$ is closed and that $S$ contains $S_{0}$. Then one has $S /(S \cap Y) \cong(Y+S) / Y \subset X / Y$ which yields that $S /(S \cap Y)$

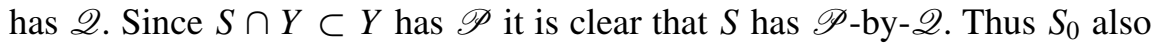
has $\mathscr{P}$-by- $\mathscr{Q}$. 
Corollary 4.2. Being an Asplund space is a 3-space property.

Proof. Having separable dual is a 3-space property which passes to subspaces.

THEOREM 4.3. The following three properties are equivalent:

(i) separable $_{*}$-by-reflexive,

(ii) separable-by-reflexive and Asplund,

(iii) (reflexive-by-separable $)_{*}$.

Proof. (i) $\Rightarrow$ (ii) This is obvious from Corollary 4.2.

(ii) $\Rightarrow$ (iii) Suppose that $Y$ is separable and $Z$ reflexive. If $X$ is Asplund, then $X^{*} / Y^{\perp} \cong Y^{*}$ is separable. Clearly $Y^{\perp} \cong Z^{*}$ and so $X^{*}$ is reflexive-byseparable.

(iii) $\Rightarrow$ (i) If $X$ has (reflexive-by-separable) $)_{*}$ then there is a reflexive (hence weak* closed) subspace $R$ of $X^{*}$ with $X^{*} / R$ separable. Clearly $X / R_{\perp}$ is reflexive since $R^{*}$ is reflexive; and $\left(R_{\perp}\right)^{*} \cong X^{*} / R$ is separable.

In [8, p. 115], a Banach space was said to have the Plichko-Valdivia property $(\mathscr{P} \mathscr{V})$ if it could be expressed as the direct sum of a reflexive subspace and a separable subspace. We prefer a different definition: we will say that a Banach space $X$ has $\mathscr{P} \mathscr{V}$ if it contains a reflexive subspace $R$ and a separable subspace $S$ so that $X=R+S$. It is shown in $\S 5$ that these two definitions are equivalent. Liftability of separability implies that $\mathscr{P} \mathscr{V}$ is equivalent to reflexive-by-separable.

Now we can give the promised counterexample to the duality problem.

THEOREM 4.4. The property $\mathscr{P} \mathscr{V}_{*}$ is a 3-space property, although $\mathscr{P} \mathscr{V}$ itself is not a 3-space property.

Proof. The first assertion follows from the facts that being Asplund and being separable-by-reflexive are both 3-space properties, whose combination is equivalent to $\mathscr{P} \mathscr{V}_{*}$. For the second, recall the Johnson-Lindenstrauss space $J L$ from Example 3.6. It is obvious that $c_{0}$ and $J L / c_{0}$ have $\mathscr{P} \mathscr{V}$. Since $J L$ is not WCG, it cannot have $\mathscr{P} \mathscr{V}$.

We have just seen that every space with $\mathscr{P} \mathscr{V}_{*}$ is Asplund. The following well-known generalization [43, Theorem 2.43] of this result will be needed several times in $\S 6$ and $\S 7$. Its proof is short enough to bear repeating.

Proposition 4.5. All $\mathrm{WCG}_{*}$ spaces are Asplund spaces. Equivalently, WCG dual spaces have the Radon-Nikodým Property.

Proof. Since the dual of a subspace is a quotient of the dual space, it suffices to establish this in the separable case. If $K \subset X^{*}$ is weakly compact, 
then the weak and weak* topologies coincide on $K$. If $X$ is separable, then $K$ is weak* metrizable, hence weakly metrizable, hence weakly separable. If $K$ generates $X^{*}$, then $X^{*}$ is separable.

\section{Projections on WCG spaces and applications}

One of the most interesting properties of WCG spaces is the so-called separable complementation property. We recall this result and some applications in this section. Except for Theorems 7.1 and 7.2, all later results in the paper depend on this section.

Weakly compactly generated spaces have many other interesting properties, for which the reader may refer to [13] or [37]. For the most part, we will only need the following result about them. (Admittedly, a somewhat stronger result was used in the proof of Theorem 3.4.) This was originally proved in [1, Lemma 4]. Various simpler proofs are now available; possibly the one in [55] is the shortest and most accessible. Another very short proof appears in [44].

THeOREM 5.1. Let $A_{0}$ be a countable subset of a WCG space $X$, and let $B_{0}$ be a countable subset of $X^{*}$. Then there is a norm one projection $P$ on $X$ with separable range, such that $A_{0} \subset P X$ and $B_{0} \subset P^{*} X^{*}$.

As well as alternative proofs, a number of extensions of this result exist in the literature [13], [21], [26], [30], [42], [53]. Here we will mention only two of them. One is the following: if $X, A_{0}, B_{0}$ are as above, and $Y$ is an arbitrary closed subspace of $X$, then there is a projection $P$ as above, with the additional property that $P Y \subset Y$. This was proved by different methods in [30] and [26], neither of them elementary. We mention this because although several results below were originally proved using this version, it is worth pointing out that every result which we know which was originally proved using $P Y \subset Y$ can now be proved without this additional conclusion.

However we learnt from a recent conversation with M. Fabian and J. Orihuela that this version can also be established by elementary methods. The basic idea is that any countable amount of information can be smuggled into the conclusion of Theorem 5.1. In particular, a simple modification of the proof shows that $P$ can be chosen to have norm one with respect to each of a countable family of equivalent norms on $X$. If $\|\cdot\|_{n}$ denotes the gauge functional of $(1 / n)$ ball $(X)+\operatorname{ball}(Y)$, and $\|P\|_{n} \leq 1$ for all $n$, a short calculation shows that $P Y \subset Y$. This argument appears in [18, p. 109], where it is used to prove a similar sort of result.

The other generalization is the following result from [51], applicable in arbitrary Banach spaces, which was motivated by earlier work in [25]. It will be needed towards the end of $\$ 7$. The technical ideas are much the same as in 
[1], with weak* compactness of the dual ball replacing weak compactness of a generating set. For a proof see [51, Proposition 2] or [24, Lemma III.4.3].

Proposition 5.2. Let $N$ be a separable subspace of $X$ and $F$ a separable subspace of $X^{*}$. Then $X$ has a separable subspace $M$ containing $N$, which admits a linear extension operator $T: M^{*} \rightarrow X^{*}$ with $T\left(M^{*}\right) \supset F$. In particular, $M^{*}$ is isometric to a complemented subspace of $X^{*}$.

If $N^{*}$ is separable, can one choose $M^{*}$ to be separable as well? This is obviously possible if $X$ is an Asplund space, but in general it is not: take $X=L_{1}(0,1)$ and let $N$ be the closed linear span of the Rademacher functions. A completely different proof that $M$ can be so chosen, under the stronger assumptions that $X / N$ is reflexive and $F=\{0\}$, appears as the Banach space case of [56, Theorem 5].

One useful corollary was promised in the last section. Again, our proof is simpler than the original, requiring no knowledge of injections into $c_{0}(\Gamma)$.

Corollary 5.3. [32]. Let $X$ be a WCG space, $Y$ a subspace with $X / Y$ separable. Then $Y$ is also WCG.

Proof. Since $Y^{\perp} \cong(X / Y)^{*}$ is weak* separable, one may choose a countable set $B$ in $X^{*}$ which is weak* dense in $Y^{\perp}$. Theorem 5.1 then gives us a projection $P$ on $X$ with separable range, and so that $Y^{\perp} \subseteq P^{*}\left(X^{*}\right)=(\text { ker } P)^{\perp}$. Obviously ker $P$ is WCG, ker $P \subseteq Y$ and $Y / \operatorname{ker} P \subseteq X / \operatorname{ker} P$ is separable. By Proposition 3.2, $Y$ is WCG.

Now we show that a Banach space $X$ is separable $\oplus$ reflexive (if and) only if it has $\mathscr{P} \mathscr{V}$. Thus our definition of $\mathscr{P} \mathscr{V}$ is consistent with that given in [8, p. 115].

Proposition 5.4. For any Banach space, the following properties are equivalent:

(i) the Plichko-Valdivia property,

(ii) reflexive $\oplus$ separable,

(iii) weakly compactly generated \& separable-by-reflexive.

Proof. It is trivial that (ii) implies (i), and (i) implies (iii) is clear from $\S 3$ and $\$ 2$. To show that (iii) implies (ii), suppose $X$ contains a separable subspace $S_{0}$ with $X / S_{0}$ reflexive. By Theorem 5.1, $S_{0}$ is contained in a complemented separable subspace $S$. The complement of $S$ is isomorphic to $X / S$ which is isomorphic to a quotient of $X / S_{0}$ which is reflexive. Thus $X / S$ is reflexive and $X$ has $\mathscr{P} \mathscr{V}$.

Although $\mathscr{P} \mathscr{V}$ is not a 3 -space property, it does pass to subspaces and quotients [45]. Now we give a new proof of this, avoiding the result used in the 
original proof that the projection in the Theorem 5.1 can be chosen to leave a given subspace invariant.

THeORem 5.5. Let $X$ be a Banach space with $\mathscr{P} \mathscr{V}$ and $Y$ a subspace of $X$. Then $Y$ and $X / Y$ also have $\mathscr{P} \mathscr{V}$.

Proof. That $X / Y$ has $\mathscr{P} \mathscr{V}$ is clear from Theorem 2.7 and Proposition 5.4. For subspaces, one needs to work a bit harder.

Write $X=R \oplus S$ where $R$ is reflexive and $S$ is separable. It is obvious that any subspace containing $S$ has $\mathscr{P} \mathscr{V}$. In particular, $X_{0}=\overline{Y+S}$ has $\mathscr{P} \mathscr{V}$, and thus is WCG. Since $X_{0} / Y$ is separable, Corollary 5.3 tells us that $Y$ is also WCG. But $Y$ is separable-by-reflexive by Theorem 2.7. By Proposition 5.4, $Y$ has $\mathscr{P} \mathscr{V}$.

Call a Banach space hereditarily WCG, or HWCG, if every closed subspace is WCG. Obviously every $\mathscr{P} \mathscr{V}$ space is HWCG. With almost no changes, the preceding proof gives us the following. We are indebted to M. Fabian for posing the question which led to this result.

THEOREM 5.6. Every (hereditarily WCG)-by-separable space is hereditarily WCG.

It has long been known that WCG Asplund spaces are HWCG ([31], [13, Corollary VI.4.4] or [18, §8.3]). In particular $c_{0}(\Gamma)$ is a HWCG space without $\mathscr{P} \mathscr{V}$. Example 3.3 shows that HWCG is not a 3-space property; we do not know whether HWCG passes to direct sums. Recalling Jarchow's problem, it would be interesting to know whether $\mathrm{HWCG}_{*}$ is a 3-space property.

Proposition 5.7. A dual space has $\mathscr{P} \mathscr{V}$ (if and) only if it is the direct sum of a reflexive space and a separable dual space. Thus every $\mathscr{P} \mathscr{V}$ dual space has a predual with $\mathscr{P} \mathscr{V}$.

Proof. Suppose that $X^{*}$ has $\mathscr{P} \mathscr{V}$. Then $X^{*}=R \oplus S$, where $R$ is reflexive and $S$ is separable. Since $R$ is weak* closed, one has $R=M^{\perp}$ for some subspace $M$ of $X$. But then $S \cong X^{*} / M^{\perp} \cong M^{*}$, so $S$ is isomorphic to a dual space. (Of course, $S$ need not be weak* closed.)

The Johnson-Lindenstrauss space shows that a $\mathscr{P} \mathscr{V}$ dual space may have a predual without $\mathscr{P} \mathscr{V}$. We conjecture that every WCG dual space has a WCG predual. 


\section{More stability results and twisted properties}

It has already been shown that WCG and $\mathscr{P} \mathscr{V}$ are not 3 -space properties. The main result of this section is that $\mathscr{P}{ }_{* *}$ implies $\mathscr{E} \mathrm{WCG}$. This means that $\mathscr{P} \mathscr{V}_{* *}$-by-WCG implies WCG, which includes a number of interesting special cases. First, a partial result.

Proposition 6.1. Every $\mathscr{P} \mathscr{V}_{* *}$-by- $\mathscr{P} \mathscr{V}$ space has $\mathscr{P} \mathscr{V}$.

Proof. Since $\mathscr{P} \mathscr{V}_{* *}$ is a 3-space property, every $\mathscr{P} \mathscr{V}_{* *}$-by-reflexive space has the property $\mathscr{P} \mathscr{V}_{* *}$, hence $\mathscr{P} \mathscr{V}$. Given our previous results, it follows that

$$
\begin{aligned}
\mathscr{P}_{V_{* *} \text {-by- } \mathscr{P} \mathscr{V}} & \Rightarrow \mathscr{P}_{* *} \text {-by-reflexive-by-separable } \\
& \Rightarrow \mathscr{P} \mathscr{V} \text {-by-separable } \\
& \Rightarrow \mathscr{P} \mathscr{V} .
\end{aligned}
$$

With essentially one exception, we have now completely answered the question: what can be said about $\mathscr{P}$-by- $\mathscr{Q}$ spaces when $\mathscr{P}, \mathscr{Q} \in$ \{separable, separable $_{* *}$, reflexive, WCG, $\left.\mathscr{P} \mathscr{V}, \mathscr{P}^{\mathscr{*}}{ }_{* *}\right\}$ ? We now plug this gap by showing that $\mathscr{P} \mathscr{V}_{* *}$-by-WCG implies WCG. (The old problem from [32, p. 229], whether $\mathrm{WCG}_{* *}$ implies WCG, still appears to be open. If this problem has a positive solution, it will be interesting to know whether $\mathrm{WCG}_{* *}$-by-WCG implies WCG. A very easy partial result, using Lemma 2.2, is that $\mathrm{WCG}_{* *}$-byreflexive implies $\mathrm{WCG}^{\mathrm{co}}$. However the dual of the James Tree space, described in Example 6.5, shows that $\mathrm{WCG}^{\mathrm{co}}$ does not imply WCG.)

The following result is so important to us that it will often be applied without mention.

Proposition 6.2. Let $\mathscr{P}$ be any Banach space property for which $\mathscr{P}$-byreflexive implies WCG. Then $\mathscr{P}$ implies $\mathscr{E} \mathrm{WCG}$.

Proof. Suppose that the subspace $Y$ has $\mathscr{P}$ and that $X / Y$ is WCG. According to Davis et al. ([10] or [18, Theorem 1.2.3]) there is a reflexive space $R$ and a continuous linear operator $T: R \rightarrow Z$ with dense range. Now we put $B=\{x \in X:\|x\| \leq 1, x+Y \in T$ (ball $R$ ) $\}$ and argue as in the proof of Theorem 3.4. Let $X_{B}$ denote the linear span of $B$ equipped with the norm whose unit ball is $B$. Then $X_{B} / Y$ is isomorphic to $R$ and thus reflexive. Since $Y$ has $\mathscr{P}, X_{B}$ must be WCG. Since $B$ is bounded, the inclusion mapping $X_{B} \rightarrow X$ is continuous and has dense range. Thus $X$ is WCG.

Of course, this gives another proof of Proposition 3.1.

COROLlary 6.3. Every $\mathscr{P}_{* *}$ space has $\mathscr{E} \mathrm{WCG}$. 
Following [41, p. 31], we call a Banach space $X$ coseparable if it has the property separable ${ }^{\text {co }}$, i.e. if $X^{* *} / X$ is separable. This is obviously a 3-space property, shared by all reflexive and all separable s** $_{*}$ spaces. The main part of the next result, namely the equivalence of (i) and (iv), shows that there are essentially no other coseparable spaces. This appeared first in [54], although the proof there seems to be incomplete. Specifically, the claim on [54, p. 13] that $F$ can be chosen so that its weak* closure contains $E^{\perp}$ is not clear to us. The proof below is a specialisation of that given in [58]. Note that results about such spaces are far from vacuous: Davis et al. [10] showed that every WCG space is isomorphic to $X^{* *} / X$ for some Banach space $X$.

THEOREM 6.4. The following Banach space properties are equivalent:

(i) separable $e^{\mathrm{co}}$,

(ii) separable $_{* *}-$ by-reflexive,

(iii) $\mathscr{P} \mathscr{V}_{* *}$ and Asplund,

(iv) reflexive $\oplus$ separable $_{* *}$.

In particular, every coseparable space has $\mathscr{P} \mathscr{V}$, and hence is WCG.

Proof. (i) $\Rightarrow$ (ii). Suppose that $X^{* *} / X$ is separable, and consider the following subspace of $X^{* *}$ containing $X$ :

$$
G=\bigcup\left\{M^{\perp \perp}: M \subset X, M \text { separable }\right\} .
$$

A moment's reflection shows that $G$ is closed. Since $G / X$ is separable, there is a sequence $\left(x_{n}\right)$ of elements of $G$ whose union with $X$ is dense in $G$. For each integer $n$, select a separable subspace $M_{n}$ of $X$ such that $x_{n} \in M_{n}^{\perp \perp}$. Denote by $N$ the separable subspace of $X$ spanned by the union of all $M_{n}$.

By Lemma 2.1, $X+N^{\perp \perp}$ is closed in $X^{* *}$. Thus, $X+N^{\perp \perp}=G$.

Now consider a bounded sequence $\left(x_{n}+N\right)$ in $X / N$. Clearly one may suppose that $\left(x_{n}\right)$ is a bounded sequence in $X$. Let $F \in X^{* *}$ be an arbitrary weak ${ }^{*}$ limit point of $\left(x_{n}\right)$. Since $\left(x_{n}\right)$ is contained in a separable subspace $M \subset X$, we must have $F \in M^{\perp \perp} \subset G$. Thus we can write $F=x+g$ where $x \in X$ and $g \in N^{\perp \perp}$. Now let $f$ be any functional in $N^{\perp}$. Then $f(x)=(x+g)(f)=F(f)$ is a limit point of the scalar sequence $f\left(x_{n}\right)=$ $f\left(x_{n}+N\right)$. Identifying $N^{\perp}$ with $(X / N)^{*}$ one sees that $\left(x_{n}+N\right)$ has a weak limit point, namely $x+N$. The Eberlein-Šmulian Theorem then implies that $X / N$ is reflexive.

By Lemma 2.1, $N^{* *} / N$ is isomorphic to a subspace of $X^{* *} / X$, and so must be separable. Thus $N^{* *}$ is also separable.

(ii) $\Rightarrow$ (iii) We have remarked that $\mathscr{P} \mathscr{V}_{* *}$ and Asplundity are both 3-space properties; obviously both are implied by separable s** $_{\text {and }}$ and reflexivity. 
(iii) $\Rightarrow$ (iv) Suppose that $X$ has $\mathscr{P}_{* *}$. By Theorem 5.5, $X$ also has $\mathscr{P} \mathscr{V}$, so one may write $X=R \oplus S$ where $S$ is separable and $R$ is reflexive. By Proposition 4.5, $X^{*}$ is Asplund. If $X$ is also Asplund, it is easy to check that every complemented separable subspace of $X$ has separable bidual. In particular, $S$ is separable $_{* *}$.

(iv) $\Rightarrow$ (i) is trivial.

For quasireflexive spaces, i.e. spaces with $X^{* *} / X$ finite dimensional, there is a shorter proof of the conclusion of Theorem 6.4(iv). Civin and Yood [9, Theorem 4.6] prove more or less by induction on $\operatorname{dim} X^{* *} / X$ that such spaces are reflexive-by-separable. Hence they have $\mathscr{P} \mathscr{V}$ and Proposition 5.4 can be applied.

Since coseparability implies $\mathscr{P}^{* *}$, we see that coseparable-by-WCG implies WCG [57, Theorem 1] and that quasireflexive-by-WCG implies WCG.

Since

$$
\begin{aligned}
& \text { separable }_{* *} \quad \Longrightarrow \quad \text { separable } \\
& \text { reflexive } \Rightarrow \text { coseparable } \Rightarrow \mathscr{P}_{* *} \Rightarrow \mathscr{P} \mathscr{V} \Rightarrow \text { WCG }
\end{aligned}
$$

we can summarize all the preceding results by saying that if $\mathscr{P}$ and $\mathscr{Q}$ are chosen amongst these seven properties, then either $\mathscr{P}$-by- $\mathscr{Q} \Rightarrow \min \{\mathscr{P}, \mathscr{Q}\}$ or $J L$ has $\mathscr{P}$-by- $\mathscr{Q}$. Of course the minimum is taken with respect to the lattice ordering induced by the preceding diagram.

The space $J L^{*}$ is an example of a space with $\mathscr{P} \mathscr{V}$ whose predual $J L$ does not have $\mathscr{P} \mathscr{V}$. The lifting property of $\ell_{1}$ is not essential to prove that $J L$ has $\mathscr{P} \mathscr{V}_{*}$; the important point, as Proposition 5.7 shows, is that $c_{0}^{*}$ is separable. Theorem 6.4 shows that the Johnson-Lindenstrauss example is "sharp". Amongst other things, it shows that if $X$ is separable ${ }_{* *}$-by-reflexive then $X^{* *}$ has $\mathscr{P} \mathscr{V}$, whence $X, X^{*}$ and $X^{* *}$ are all WCG.

This leads us to ask whether $\mathscr{P} \mathscr{V}_{* *}$ can be replaced by $\mathrm{WCG}_{* *}$ in Theorem 6.4(iii). It cannot; a simple counterexample is the $\ell_{2}$ direct sum of uncountably many copies of James's quasireflexive space.

The following example, the James Tree space, shows that $\mathscr{P} \mathscr{V}_{* *}$ is a strictly weaker property than coseparability. Thus the words "and Asplund" cannot be deleted from part (iii) of Theorem 6.4. In connection with this part of the proof, note that the property (separable $e_{* *}$ ) ${ }^{\text {sep }}$ is equivalent to the property (Asplund \& Asplund ${ }_{*}$ ). Since we don't need this result, which depends on Proposition 5.2, we omit the proof.

Example 6.5. There is a separable dual space, denoted $J T$, such that $J T^{* *} / J T$ is a non-separable Hilbert space. 
DESCRIPTION. This space was first constructed in [27], then studied further in [38]. The essential properties [38] are that it is a separable dual space, and that $J T^{* *} / J T$ is a non-separable Hilbert space. (Note that the existence of a separable dual space $Y$, such that $Y^{* *} / Y$ is non-separable and reflexive, is also proved in [10, pp. 324-325].) It seems to be common practice to denote its predual $J T_{*}$ by $B$. Clearly $\left(B^{* *} / B\right)^{*} \cong J T^{* *} / J T$ is also a non-separable Hilbert space, and so $B^{* *}$ is not separable. Thus $B^{*}$ is not an Asplund space (even though it does not contain $\ell_{1}$ ), and Proposition 4.5 implies that $B^{* *}$ is not WCG. This gives another counterexample to the 3 -space problem for WCG spaces. In particular, $B$ is not complemented in $B^{* *}$, and so $B$ is not a dual space.

Since $\mathscr{P} \mathscr{V}_{* *}$ passes to subspaces, we see that any extension of a subspace of $J T$ by a WCG space is WCG. More generally, any separable coreflexive space which is complemented in its bidual has $\mathscr{P} \mathscr{V}_{* *}$, and thus $\mathscr{E} \mathrm{WCG}$.

It is natural to ask what conclusion can be drawn when some higher dual of $X$ has $\mathscr{P} \mathscr{V}$. Obviously $\left\{n:\right.$ the $n^{\text {th }}$ dual of $X$ has $\left.\mathscr{P} \mathscr{V}\right\}$ is closed under subtraction by 2 , but nothing more can be said. For $B$ this set is precisely the union of 0 and the odd integers; for $J T$ it is precisely the even integers. Direct summing with other examples then shows that any set which is closed under subtraction by 2 can be realised as $\left\{n:\right.$ the $n^{\text {th }}$ dual of $X$ has $\left.\mathscr{P} \mathscr{V}\right\}$, for some Banach space $X$.

Since $\mathscr{P} \mathscr{V}$ kindly provided us with an example of a non-3-space property whose dual is a 3 -space property, it is natural to ask if it provides a counterexample for the (still open) problem: if $\mathscr{P}^{\mathrm{co}}$ is a 3 -space property, must $\mathscr{P}$ be a 3-space property? It does not, as the James Tree now helps us see.

EXAMPLE 6.6. The properties $\mathscr{P} \mathscr{V}^{\mathrm{co}}$ and $\mathrm{WCG}^{\mathrm{co}}$ are not 3 -space properties.

Proof. Let $B=J T_{*}$ be the predual of the James Tree space. Applying [10] one finds a Banach space $X$ with $X^{* *} / X \cong B$. Since $X^{* *} / X \cong B$ is separable, one sees that $X$ has $\mathscr{P} \mathscr{V}^{\text {co }}$. Since $B^{* *} / B$ is a Hilbert space, $X^{* *} / X$ also has $\mathscr{P} \mathscr{V}^{\text {co }}$. However $X^{* * * *} / X^{* *} \cong\left(X^{* *} / X\right)^{* *} \cong B^{* *}$ is not WCG, and thus $X^{* *}$ does not have $\mathscr{P} \mathscr{V}^{\text {co }}$.

Note that $\mathscr{P} \mathscr{V}^{\text {co }}$ does not imply $\mathscr{P} \mathscr{V}$. In fact the space $J T^{*}$ shows that reflexive ${ }^{\text {co }}$ does not even imply WCG.

\section{Duality and new stability results}

The main result of the last section was that $\mathscr{P} \mathscr{V}_{* *}$, which is a 3 -space property, implies $\mathscr{E} \mathrm{WCG}$. In this section we present several other properties which also imply $\mathscr{E}$ WCG, in particular the combination (WCG\&Asplund\&dual), and being a subspace of a space with $(\mathscr{P} \mathscr{V} \& \mathscr{C} \mathscr{B})$. These are probably not 3 -space 
properties; nevertheless any finite twisted sum of Banach spaces, in which each of the components has one of these properties, also has $\mathscr{E} \mathrm{WCG}$.

Call a Banach space a dual space if it is isomorphic to the dual of another Banach space. A little care is needed here, since any non-reflexive space can be renormed so as to be not isometric to the dual of any space [11]. It has recently been shown [5, Theorem 1 or 5] that being a dual space is not a 3 -space propery. The following partial result is quite sufficient for us. It is needed for all the results in this section, and we present a new proof.

THEOREM 7.1. [14]. Every extension of a dual space by a reflexive space is a dual space, and the dual subspace is weak* closed.

Proof. Let $Y$ be a dual space with $X / Y$ reflexive. Let $R: X^{*} \rightarrow Y^{*}$ be the restriction map, and set $W=R^{-1}\left(Y_{*}\right)$, where $Y_{*}$ is an isomorphic predual of $Y$. We claim that $X \cong W^{*}$; it suffices to show that every bounded net in $X$ has a $\sigma(X, W)$ convergent subnet. So let $\left(x_{\alpha}\right)$ be any bounded net in $X$. Using Lemma 2.1(ii) and the reflexivity of $X / Y$ we may suppose (passing to a subnet if necessary) that there is an $x \in X$ and an $F \in Y^{\perp \perp}$ so that $x_{\alpha} \rightarrow x+F$ weak*. Of course there is a bounded net in $Y$ which converges weak* to $F$. The standard trick of expanding the index sets permits us to assume that this net is defined on the same index set as $\left(x_{\alpha}\right)$. Thus we may write $y_{\alpha} \rightarrow F$ weak*. It clearly follows that $x_{\alpha}-y_{\alpha} \rightarrow x$ weakly. Since $Y$ is a dual space, we may (passing to a subnet if necessary) assume that $y_{\alpha} \rightarrow y$ for the topology $\sigma\left(Y, Y_{*}\right)$ and some $y \in Y$. Then for any $f \in W$ we have $f\left(x_{\alpha}-y_{\alpha}\right) \rightarrow f(x)$ and $f\left(y_{\alpha}\right) \rightarrow f(y)$. This implies that $x_{\alpha} \rightarrow x+y$ with respect to $\sigma(X, W)$, as required.

Trivially $Y^{\perp} \subset W$, and so the $\sigma(X, W)$ closure of $Y$ is contained in the $\sigma\left(X, Y^{\perp}\right)$ closure of $Y$. But the latter is simply $Y$, which is thus $\sigma(X, W)$ closed.

The hypothesis of reflexivity can be weakened considerably. According to [5, Proposition 3], it suffices to assume (in the notation of the preceding proof) that $X / Y \cong W^{*}$ for some Banach space $W$ with the properties that $W$ is complemented in its bidual, and $W^{* *} / W$ is injective. In particular, every dual-by-quasireflexive space is a dual space.

THEOREM 7.2. Every (dual \& $\mathscr{P} \mathscr{V}$ ) space enjoys $\mathscr{E} \mathrm{WCG}$. In particular, $\ell_{1}$ enjoys $\mathscr{E} \mathrm{WCG}$.

Proof. Thanks to Proposition 6.2, it suffices to prove that every (dual $\mathscr{P} \mathscr{V}$ )by-reflexive space is WCG. Consider a $\mathscr{P} \mathscr{V}$ dual space $Y$ with $Z$ reflexive. By Theorem $7.1 X$ is a dual space; in the predual $X_{*}$, note that the subspace $Z^{*}$ and the quotient $Y_{*} \cong X_{*} / Z^{*}$ enjoy the 3 -space property $\mathscr{P}_{*}$. Hence $X$ has $\mathscr{P} \mathscr{V}$. 
Note that Theorem 7.2 does not depend on Theorem 5.1, since we used only the trivial implication $\mathscr{P} \mathscr{V} \Rightarrow$ reflexive-by-separable. Note also that the second part of this result is not trivial, since there are non-trivial twisted sums of $\ell_{1}$ and $\ell_{p}(\Gamma)$, for $1<p<\infty$. This follows from [35, $\left.\$ 4\right]$ when $\Gamma$ is countably infinite, and the general case then follows by forming a direct sum with $\ell_{p}(\Gamma)$.

As a quick application, we give a simple proof of a result from [4]. The action takes place in $J H^{*}$, the dual of the James-Hagler space [22], and concerns the subspace $F$ of $J H^{*}$ generated by the evaluation functionals. According to [4, Lemma 5], $F$ fails the Radon-Nikodým Property, and so cannot be a dual space. We prove the latter fact directly. Clearly $F$ is separable, and it can be shown that $J H^{*} / F \cong c_{0}(\Gamma)$. Since $J H$ is separable but $J H^{*}$ is not, Proposition 4.5 shows that $J H^{*}$ cannot be WCG. Thus we have another counterexample to the 3-space problem for WCG spaces. By Theorem 7.2, $F$ cannot be a dual space. We note also that every infinite-dimensional subspace of $J H^{*}$, in particular of $F$, contains $\ell_{1}$ [22, Theorem 1(e), p. 304]. So it is not true that (separable \& $\ell_{1}$-saturated)-by-WCG implies WCG.

A short argument, using Corollary 5.3, now shows that every subspace of a $\mathscr{P} \mathscr{V}$ dual space enjoys $\mathscr{E} \mathrm{WCG}$. This obviously includes Corollary 6.3. We will not prove this now, as a much stronger result appears later.

First, let us consider some questions which arise naturally from Theorem 7.2.

QUESTION A. , In Theorem 7.2, can “ $\mathscr{P} \mathscr{V}$ ” be replaced by “WCG”? Equivalently, is $W C G_{*}$ a 3 -space property? Does reflexive-by- $\mathrm{WCG}_{*}$ imply $\mathrm{WCG}_{*}$ ? Does (WCG dual)-by-reflexive imply WCG?

QUESTION B. , If so, can "dual” then be replaced by "RNP”? Equivalently, is (WCG \& RNP) a 3-space property?

Question C. , In Theorem 7.2, can “dual” be replaced by “ $\mathscr{C} \mathscr{B}$ ”?

Question D., In Theorem 7.2, can $\ell_{1}$ be replaced by $L_{1}(\mu)$, for a $\sigma$-finite measure $\mu$ ?

Question E. , Does $\mathscr{E} \mathrm{WCG}$ pass to subspaces?

We can give partial answers to some of these questions. Let us discuss them in turn before proving anything.

A. Corollary 7.7 asserts that " $\mathscr{P} \mathscr{V}$ " can be replaced by "WCG \& Asplund". This includes the case of an $\ell_{2}$ product of uncountably many copies of James's space, which does not enjoy $\mathscr{P} \mathscr{V}$. Of course many WCG dual spaces, including $J L^{*}, J T$ and all of its even duals, $\ell_{1}$ and all reflexive spaces do have $\mathscr{P} \mathscr{V}$. 
A positive answer to Question A would give another response to Jarchow's request for a non-3-space property whose dual is a 3-space property. An example is given in [5, Theorem 5] of an extension of a separable Hilbert space by a separable dual space which is not a dual space. This shows that neither (separable \& dual) nor (WCG \& dual) is a 3-space property, and thus the most obvious method for resolving this problem is not available.

B. We have no idea about this question, which obviously arises from Proposition 4.5. Note that $\mathrm{WCG}_{*}$ and (WCG\&RNP) $)_{*}$ are the same property. A negative answer to Question A would imply the existence of an example with $Y$ a WCG dual space, $Z=R$ reflexive and yet $X$ not WCG. We emphasize that, by the previous results, neither $Y_{*}$ nor $R$ could be separable. We will see shortly that $Y$ could not even be a subspace of a $\mathscr{P} \mathscr{V}$ dual space. In each counterexample to the 3 -space property for WCG spaces known to us the subspace is separable and contains either $c_{0}$ or $B=J T_{*}$ or $F$ (the subspace of $J H^{*}$ discussed earlier), none of which has the Radon-Nikodým Property.

Recall that a Banach space is said to have the point of continuity property (PCP) if every non-empty closed bounded subset admits a point where the identity mapping is weak-to-norm continuous. Clearly this is implied by the RNP; the two are equivalent in subspaces of $L_{1}$ spaces [4]. We note that, by [16, p. 346] and [4] respectively, the spaces $B$ and $F$ have PCP. Thinking about $J T^{*}$ and $J H^{*}$ again, we see that (separable \& PCP)-by-WCG does not imply WCG.

Not every separable space with the Radon-Nikodým Property embeds into a separable dual space ([3], [39], [33]), so it may be of interest to know that every extension of a subspace of a $\mathscr{P} \mathscr{V}$ dual space by a WCG space is WCG. At first sight, it appears to be a mild generalization of Theorem 7.2, but it actually lies somewhat deeper, since it depends on Theorem 5.1. We will not prove this now, as a stronger statement appears in Theorem 7.6. The techniques needed are the same as those needed for the coming partial solution of questions $\mathrm{C}$ and D.

C. Actually Theorem 7.6 gives a complete positive answer to this question.

D. Corollary 7.4 shows that this question has a positive answer for separable $L_{1}$ spaces. The general question is obviously equivalent to: if $\mu$ is a $\sigma$-finite measure, does $L_{1}(\mu)$-by-reflexive imply WCG? This is interesting, because both reflexive spaces and such $L_{1}(\mu)$ spaces are strongly WCG in the sense of [50]. It seems to be unknown whether being strongly WCG is a 3-space property.

E. The sufficient condition defined in Theorem 7.6 obviously passes to subspaces; we do not know whether this is true for that of Corollary 7.7. Thanks to Rosenthal's counterexample to the heredity problem for WCG spaces [49], questions $\mathrm{D}$ and $\mathrm{E}$ cannot both have positive solutions. 
Now for some proofs. Just for this section, let $\mathscr{C} \mathscr{D} \mathscr{S}$ denote the property "complemented in the dual of a separable space".

Proposition 7.3. Let $Y$ be a separable space which is also $\mathscr{C} \mathscr{D} \mathscr{S}$. Then every extension of $Y$ by a reflexive space is (separable $\& \mathscr{C} \mathscr{D} \mathscr{S}) \oplus$ reflexive. Thus $Y$ enjoys $\mathscr{E} W C G$.

Proof. If $Y$ is $\mathscr{C} \mathscr{D} \mathscr{S}$, then there is a separable space $S$ and another Banach space $C$ with $S^{*}=Y \oplus C$. Let $X$ contain $Y$ with $X / Y$ reflexive. Put $X_{1}=$ $X \oplus C$. Clearly $X_{1}$ is an extension of $S^{*}$ by a reflexive space and so Theorem 7.1 gives us a reflexive-by- $S$ space $V$ with $V^{*}=X_{1}$. More precisely, let $R_{1}=\left(S^{*}\right)^{\perp}$ be the polar of $S^{*}$ calculated in $V$; then $R_{1}$ is a reflexive subspace of $V$ with $V / R_{1} \cong S$ and $S^{*}$ is weak* closed in $V^{*}$.

We claim that there is a separable subspace $W$ in $V$ and a reflexive subspace $R \subseteq R_{1}$ such that $V=W \oplus R$. Since $S^{*}$ is a weak* separable subspace of $V^{*}$, Theorem 5.1 gives us a projection $P$ on $V$ with separable range so that $P^{*}\left(V^{*}\right) \supseteq S^{*}$. Put $W=P(V)$ and $R=\operatorname{ker} P$. Then $R^{\perp}=P^{*}\left(V^{*}\right) \supseteq S^{*}=$ $R_{1}^{\perp}$, and so $R \subseteq R_{1}$ as required.

Then $X_{1}=W^{\perp} \oplus R^{\perp}$ and $W^{*} \cong R^{\perp} \supseteq S^{*}$. Since $S^{*}$ is weak* closed, we see that $R^{\perp} / S^{*}$ is isomorphic to the dual of some necessarily separable subspace of $W$ (namely $R_{1} \cap W$ ). But $R^{\perp} / S^{*} \subseteq X_{1} / S^{*}$ is also reflexive and so must itself be separable.

Recall that $Y \oplus C=S^{*} \subseteq R^{\perp} \subseteq X_{1}$. Since $C$ is complemented in $X_{1}$, it is certainly complemented in $R^{\perp}$. So we may write $R^{\perp}=U \oplus C$ for some subspace $U$ containing $Y$. Separability of $R^{\perp} / S^{*} \cong U / Y$ and of $Y$ then implies that $U$ is separable. Of course $U$ is complemented in $R^{\perp} \cong W^{*}$. Finally we see that $X \cong X_{1} / C \cong W^{\perp} \oplus R^{\perp} / C \cong R^{*} \oplus U$ is reflexive $\oplus$ (separable\& $\mathscr{C} \mathscr{D} \mathscr{S}$ ).

The final conclusion follows from Proposition 6.2.

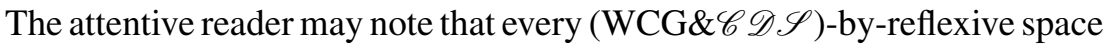

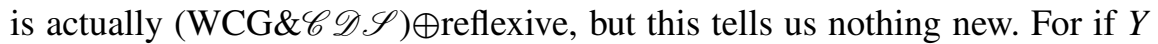
embeds in the dual of a separable space, then $Y^{*}$ will be weak* separable, and every weakly compact subset of $Y$ will be separable.

Corollary 7.4. $L_{1}(0,1)-b y-W C G$ implies WCG.

Proof. Recall that $L_{1}(0,1)$ is (isometric to) a complemented subspace of $C[0,1]^{*}$.

Proposition 7.3 shows that if $Y$ is (separable $\& \mathscr{C} \mathscr{D} \mathscr{S}$ ), then it has $\mathscr{E} \mathrm{WCG}$. This rather weak result does not even include Proposition 3.1. Now we remedy this deficiency by weakening further the conditions placed on $Y$, thus generalizing both Corollary 6.3 and Proposition 7.3. More precisely, we will replace

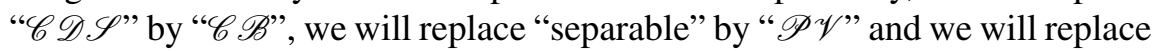


"Let $Y$ be a" with "Let $Y$ be a subspace of a". It will soon be evident that these three substitutions must be performed in this order.

It is a well known easy exercise that any Banach space which is complemented in some dual space must have $\mathscr{C} \mathscr{B}$. Thus $\mathscr{C} \mathscr{D} \mathscr{S}$ implies $\mathscr{C} \mathscr{B}$; our next result establishes the converse to this, for separable spaces.

LEMMa 7.5.

(i) Every separable Banach space with $\mathscr{C} \mathscr{B}$ is complemented in the dual of a separable space.

(ii) Every $(\mathscr{P} \mathscr{V} \& \mathscr{C} \mathscr{B})$-by-reflexive space has $\mathscr{P} \mathscr{V}$.

Proof. (i) Suppose that $F$ is separable and complemented in some dual space $X^{*}$. (We could suppose that $X=F^{*}$, but this is not necessary.) Applying Proposition 5.2 with $N=\{0\}$ gives us a separable subspace $M \subset X$ and a linear extension operator $T: M^{*} \rightarrow X^{*}$ such that $T\left(M^{*}\right) \supset F$. Since $F$ is complemented in $X^{*}$, it is undoubtedly complemented in $T\left(M^{*}\right)$, which is isomorphic to the dual of a separable space.

(ii) It is easily seen that any space with $(\mathscr{P} \mathscr{V} \& \mathscr{C} \mathscr{B})$ must be the direct sum of a reflexive space and a separable space with $\mathscr{C} \mathscr{B}$. The conclusion then follows from part (i) and Proposition 7.3.

Now we can present our main generalization of Theorem 7.2.

THEOREM 7.6. Let $Y$ be a subspace of a space with $\mathscr{P} \mathscr{V}$ and $\mathscr{C} \mathscr{B}$. Then $Y$ enjoys $\mathscr{E} \mathrm{WCG}$.

Proof. It suffices to show that $Y$-by-reflexive implies $\mathscr{P} \mathscr{V}$.

Let $R \oplus S$ be a $(\mathscr{P} \mathscr{V} \& \mathscr{C} \mathscr{B})$ space containing $Y$. It follows from the notation that $R$ is reflexive and that $S$ is separable with $\mathscr{C} \mathscr{B}$. Put $Y_{1}=\overline{Y+S}$; then $Y_{1} / Y$ is separable. Clearly $S$ is complemented in $Y_{1}$, with reflexive complement, so $Y_{1}$ is a $(\mathscr{P} \mathscr{V} \& \mathscr{C} \mathscr{B})$ space.

Now let $X$ be any extension of $Y$ by a reflexive space. Let $X_{1}$ be the pushout $\left(X \oplus Y_{1}\right) / \Delta$, where $\Delta=\{(y, y): y \in Y\}$. Then $X_{1} / Y_{1} \cong X / Y$ is reflexive, so Lemma 7.5 (ii) tells us that $X_{1}$ has $\mathscr{P} \mathscr{V}$. According to Theorem 5.5, $X$ also has $\mathscr{P} \mathscr{V}$.

Note that there are Banach spaces $Y$ satisfying the hypotheses of Theorem 7.6 which are not $\mathscr{C} \mathscr{B}$. For example, consider the kernel of a quotient mapping from $\ell_{1}$ onto $L_{1}(0,1)$ : according to [36] this space is not $\mathscr{C} \mathscr{B}$.

For another sufficient condition for $\mathscr{E} \mathrm{WCG}$, recall that Deville and Godefroy [12, p. 192] proved that (Asplund \& WCG) $)_{*}$ is a 3 -space property, thus unwittingly answering Jarchow's question. (The existence of $J L$ shows that 
(Asplund \& WCG) is not a 3-space property.) Combining this with Proposition 6.2 and Theorem 7.1 immediately gives the promised partial solution to Question A.

Corollary 7.7. Every WCG Asplund dual space enjoys $\mathscr{E} \mathrm{WCG}$.

We finish this section by asking: does $L_{2}\left(\mu, \ell_{1}\right)$ enjoy $\mathscr{E}$ WCG? This Banach space is certainly WCG but (if $L_{2}(\mu)$ is not separable) it does not satisfy any of the preceding properties.

\section{Separably distinguished spaces and related properties}

We have seen that the properties reflexive $\oplus$ separable and reflexive $\oplus$ separable $_{* *}$ are interesting. It seems natural to investigate the property reflexive $\oplus$ separable $_{*}$. This has already been done by Edgar and Wheeler [16], who called such spaces separably distinguished. Actually their definition of separably distinguished [16, p. 321] was that $X^{*}$ should contain a separable subspace $S$ for which $S^{\perp} \subseteq X$. This can only occur if $S^{\perp}$ is reflexive, so that definition is equivalent to reflexive-by-separable ${ }_{*}$. Several of the equivalences in the next result come from [16, Theorem 4.3].

THEOREM 8.1. The following Banach space properties are equivalent:

(i) reflexive $\oplus$ separable $_{*}$,

(ii) reflexive-by-separable ${ }_{*}$, i.e. separably distinguished,

(iii) reflexive-by-separable and Asplund,

(iv) $\mathscr{P} \mathscr{V}$ and Asplund,

(v) $\mathscr{P} \mathscr{V}$ and $\mathscr{P} \mathscr{V}_{*}$.

Proof. The equivalence of the two definitions of $\mathscr{P} \mathscr{V}$ ensures that (iv) $\Rightarrow$ (i). The implications (i) $\Rightarrow$ (v) and (iv) $\Rightarrow$ (ii) are trivial.

Theorem 4.3 shows that (v) $\Rightarrow$ (iv). Since Asplundity is a 3-space property, we see that (ii) $\Rightarrow$ (iii). Of course Proposition 5.4 ensures that (iii) $\Rightarrow$ (iv).

We note that neither $\mathscr{P} \mathscr{V}$, nor $\mathscr{P} \mathscr{V}_{*}$ nor Asplund alone imply that a space is separably distinguished. Suitable counterexamples are $\ell_{1}, J L$ and $c_{0}(\Gamma)$ respectively. The Johnson-Lindenstrauss space also shows that being separably distinguished is not a 3-space property.

Recall that separable ${ }_{*}$-by-reflexive is equivalent to $\mathscr{P} \mathscr{V}_{*}$. Thus we have now considered all twisted properties formed from the collection \{reflexive, separable, separable s $_{*}$, separable s $\left._{* *}\right\}$. We finish by considering a couple more properties which are stronger than being separably distinguished. 
Recall that a topological space is Polish if it is homeomorphic to a complete separable metric space. We will call a Banach space Polish if its unit ball is Polish in the weak topology.

There are various forgettable definitions of $\check{C}$ ech complete topological spaces. The unit ball of a Banach space is Čech complete in the weak topology if and only if it is a weak* $G_{\delta}$ subset of the unit ball of the bidual [16, Proposition 2.3]; in this case we will call the Banach space $\check{C} e c h$. We summarize the main results about these properties in the following result.

THEOREM 8.2.

(i) $[16$, Theorem A] Being Polish is equivalent to being separable, Asplund and having the point-of-continuity property.

(ii) [8, Chapter 4] Having PCP is a 3-space property, and thus being Polish is a 3-space property.

(iii) $[16$, Theorem B] The properties $\check{C}$ ech, reflexive-by-Polish and reflexive $\oplus$ Polish are all equivalent. In particular, Polish and Čech spaces are separably distinguished.

(iv) [16, p. 327] Thanks to the James Tree space, being Čech is not a 3-space property.

(v) [16, Theorem 4.5] The property Čech ${ }_{*}$ is equivalent to coseparability and is thus a 3-space property.

(vi) [16, Example 4.8] The properties Polish and Čech do not pass to quotient spaces.

(vii) [16, Proposition 4.2] If $Y$ is a reflexive subspace of $X$, then $X$ is $\check{C}$ ech if and only if $X / Y$ is Čech.

Obviously (iv) and (v) imply that being Čech provides yet another counterexample for Jarchow's problem. Example 6.6 also shows that $\mathrm{Cech}^{\mathrm{co}}$ is not a 3 -space property.

In connection with a variant of Question $\mathrm{C}$ from the previous section, $J T^{*}$ shows us that Polish-by-reflexive does not imply WCG. However being Polishby-reflexive is a 3-space property, according to Proposition 2.4 and Theorem 8.2(iii).

ACKNOWLEDGEMENTS. The first and second authors were supported in part by DGICYT Projects PB94-1052 and PB97-0377. Part of this work arose from discussions between the third and fourth authors at the 2nd International Workshop on Functional Analysis at the University of Trier in September 1997. The fourth author is indebted to the Humboldt Foundation for supporting his participation at this meeting. 


\section{REFERENCES}

1. Amir, D. and Lindenstrauss, J., The structure of weakly compact sets in Banach spaces, Ann. of Math. 88 (1968), 35-46.

2. Argyros, S., Weakly Lindelöf determined Banach spaces not containing $\ell_{1}$, preprint.

3. Bourgain, J. and Delbaen, F., A class of special $\mathscr{L}_{\infty}$ spaces, Acta Math. 145 (1980), 155-176.

4. Bourgain, J. and Rosenthal, H. P., Geometrical implications of certain finite dimensional decompositions, Bull. Soc. Math. Belg. 32 (1980), 57-82.

5. Cabello Sánchez, F. and Castillo, J. M. F., Duality and twisted sums of Banach spaces, J. Funct. Anal., 175 (2000), 1-16.

6. Cabello Sánchez, F. and Castillo, J. M. F., Uniform boundedness and twisted sums of Banach spaces, submitted.

7. Castillo, J. M. F. and González, M., The Dunford-Pettis property is not a three space property, Israel J. Math. 81 (1993), 297-299.

8. Castillo, J. M. F. and González, M., Three space problems in Banach space theory, Lecture Notes in Math. 1667 (1997).

9. Civin, P. and Yood, B., Quasi-reflexive spaces, Proc. Amer. Math. Soc. 8 (1957), 906-911.

10. Davis, W. J., Figiel, T., Johnson, W. B. and Pełczyński, A., Factoring weakly compact operators, J. Funct. Anal. 17 (1974), 311-327.

11. Davis, W. J. and Johnson, W. B., A renorming of non-reflexive Banach spaces, Proc. Amer. Math. Soc. 37 (1973), 486-488.

12. Deville, R. and Godefroy, G., Some applications of projective resolutions of identity, Proc. London Math. Soc. 67 (1993), 183-199.

13. Deville, R., Godefroy, G. and Zizler, V., Smoothness and renormings in Banach spaces, Pitman Monographs Surveys Pure Applied Math. 64, 1993.

14. Díaz, J. C., Dierolf, S., Domański, P. and Fernández, C., On the three space problem for dual Fréchet spaces, Bull. Polish Acad. Sci. 40 (1992), 221-224.

15. Diestel, J. and Uhl Jr., J. J., Vector Measures, Math Surveys Monographs 15, 1977.

16. Edgar, G. A. and Wheeler, F., Topological properties of Banach spaces, Pacific. J. Math. 115 (1984), 317-350.

17. Enflo, P., Lindenstrauss, J. and Pisier, G., On the "Three space problem", Math. Scand. 36 (1975), 199-210.

18. Fabian, M. J., Gâteaux differentiability of convex functions and topology. Weak Asplund spaces, Wiley-Interscience, New York, 1997.

19. González, M. and Onieva, V. M., Lifting results for sequences in Banach spaces, Math. Proc. Cambridge Philos. Soc. 105 (1989), 117-121.

20. Groves, J. R. J., Finitely presented centre-by-metabelian groups, J. London Math. Soc. 18, (1978), 65-69.

21. Gulko, S. P., The structure of spaces of continuous functions and their complete paracompactness (Russian), Uspehi Mat. Nauk 34:6 (1979), 33-40; English transl. Russian Math. Surveys 34:6 (1979), 36-44.

22. Hagler, J., A counterexample to several questions about Banach spaces, Studia Math. 60 (1977), 289-308.

23. Hall, P., Finiteness conditions for soluble groups, Proc. London Math. Soc. 4 (1954), 419-436.

24. Harmand, P., Werner, D. and Werner, W., M-ideals in Banach spaces and Banach algebras, Lecture Notes in Math. 1547 (1993).

25. Heinrich, S. and Mankiewicz, P., Applications of ultrapowers to the uniform and Lipschitz classification of Banach spaces, Studia Math. 73 (1982), 225-251.

26. Henson, C. W., The isomorphism property in nonstandard analysis and its use in the theory of Banach spaces, J. Symbolic Logic, 39 (1974), 717-731. 
27. James, R. C., A separable somewhat reflexive space with nonseparable dual, Bull. Amer. Math. Soc. 80 (1974), 738-743.

28. Jameson, G. J. O., Topology and normed spaces, Chapman and Hall, London, 1974.

29. Jarchow, H., The three space problem and ideals of operators, Math. Nachr. 119 (1984), 121-128.

30. John, K. and Zizler, V., Projections in dual weakly compactly generated Banach spaces, Studia Math. 49 (1973), 41-50.

31. John, K. and Zizler, V., Smoothness and its equivalents in weakly compactly generated Banach spaces, J. Funct. Anal. 15 (1974), 1-11.

32. Johnson, W. B. and Lindenstrauss, J., Some remarks on weakly compactly generated Banach spaces, Israel J. Math. 17 (1974), 219-230 and 32 (1979), 382-383.

33. Johnson, W. B. and Lindenstrauss, J., Examples of $\mathscr{L}_{1}$ spaces, Ark. Mat. 18 (1980), 101-106.

34. Kalton, N. J. and Peck, N. T., Twisted sums of sequence spaces and the three space problem, Trans. Amer. Math. Soc. 255 (1979), 1-30.

35. Kalton, N. J. and Pełczyński, A., Kernels of surjections from $\mathscr{L}_{1}$-spaces with an application to Sidon sets, Math. Ann. 309 (1997), 135-158.

36. Lindenstrauss, J., On a certain subspace of $\ell_{1}$, Bull. Acad. Polon. Sci. 12 (1964), 539-542.

37. Lindenstrauss, J., Weakly compact sets - their topological properties and the Banach spaces they generate, Ann. Math. Studies 69 (1972), 235-273.

38. Lindenstrauss, J. and Stegall, C., Examples of separable spaces which do not contain $\ell^{1}$ and whose duals are non-separable, Studia Math. 54 (1975), 81-105.

39. McCartney, P. W. and O'Brien, R. C., A separable Banach space having the Radon-Nikodym property that is not isomorphic to a subspace of a separable dual, Proc. Amer. Math. Soc. 78 (1980), 40-42.

40. Nakamura, M. and Kakutani, S., Banach limits and the Čech compactification of an uncountable discrete set, Proc. Imp. Acad. Japan, 19 (1943), 224-229.

41. Onieva, V. M., Notes on Banach ideals, Math. Nachr. 126 (1986), 27-33.

42. Orihuela, J. and Valdivia, M., Projective generators and resolutions of identity in Banach spaces, Rev. Mat. Univ. Complut. Madrid 2, núm. suppl., (1989), 179-199.

43. Phelps, R. R., Convex functions, monotone operators and differentiability, Lecture Notes in Math., 1364 (1989).

44. Plichko, A. M., Existence of a bounded M-basis in every WCG space (Russian), Teor. Funktsiǔ Funktsional Anal. Priložen (Kharkov), 32:4 (1979), 61-69.

45. Plichko, A. M., Some properties of Johnson-Lindenstrauss space, Funkt. Anal. i Priloz. 15 (1981), 88-89; English transl. Funct. Anal. Appl. 15 (1981), 149-150.

46. Plichko, A. M., Decomposition of Banach space into a direct sum of separable and reflexive subspaces and Borel maps, Serdica Math. J., 23 (1997), 335-350.

47. Reif, J., A note on Markuševič bases in weakly compactly generated Banach spaces, Comment. Math. Univ. Carolin. 15 (1974), 335-340.

48. Rosenthal, H. P., On relatively disjoint families of measures, with some applications to Banach space theory, Studia Math. 37 (1970), 13-36.

49. Rosenthal, H. P., The heredity problem for weakly compactly generated Banach spaces, Compositio Math. 28 (1974), 83-111.

50. Schlüchtermann, G. and Wheeler, R. F., On strongly WCG Banach spaces, Math. Z. 199 (1988), 387-398.

51. Sims, B. and Yost, D., Banach spaces with many projections, Proc. Centre Math. Appl. Austral. Nat. Univ. 14 (1986), 335-342. (In: Jefferies, B., McIntosh, A. \& Ricker, W., editors, Miniconference on Operator Theory and Differential Equations, Sydney, September 1986).

52. Stegall, C., The Radon-Nikodým property in conjugate Banach spaces, Trans. Amer. Math. Soc. 206 (1975), 213-223. 
53. Stegall, C., A proof of the theorem of Amir and Lindenstrauss, Israel J. Math. 68 (1989), 185-192.

54. Valdivia, M., On a class of Banach spaces, Studia Math. 60 (1977), 11-13.

55. Valdivia, M., Espacios de Fréchet de generación débilmente compacta, Collect. Math. 38 (1987), 17-25.

56. Valdivia, M., Resolution of identity in certain metrizable locally convex spaces, Rev. Real Acad. Cienc. Exact Fis. Natur. Madrid 83 (1989), 75-96.

57. Valdivia, M., Some properties of weakly countably determined Banach spaces, Studia Math. 93 (1989), 137-144.

58. Valdivia, M., Topological direct sum decompositions of Banach spaces, Israel J. Math. 71 (1990), 289-296.

59. Yost, D., Asplund spaces for beginners, Acta Univ. Carolin. Math. Phys. 34 (1993), 159-177.

60. Yost, D., The Johnson-Lindenstrauss space, Extracta Math. 12 (1997), 185-192.

DEPARTAMENTO DE MATEMÁTICAS

UNIVERSIDAD DE EXTREMADURA

AVDA. DE ELVAS, S/N.

06071 BADAJOZ

SPAIN

E-mail: castillo@unex.es

DEPARTMENT OF MATHEMATICS

PEDAGOGICAL UNIVERSITY

STR. SHEVCHENKO 1

316050 KIROVOGRAD

UKRAINE

E-mail: aplichko@kspu.kr.ua
DEPARTAMENTO DE MATEMÁTICAS

UNIVERSIDAD DE CANTABRIA

AVDA. LOS CASTROS, S/N.

39071 SANTANDER

SPAIN

E-mail: gonzalem@ccaix3.unican.es

DEPARTAMENTO DE MATEMÁTICAS

UNIVERSIDAD DE EXTREMADURA

AVDA. DE ELVAS, S/N.

06071 BADAJOZ

SPAIN

E-mail: dyost@unex.es

and

DEPARTMENT OF MATHEMATICS

KING SAUD UNIVERSITY

P.O. BOX 2455

RIYADH 11451

SAUDI ARABIA

E-mail: dthoyost@ksu.edu.sa 